\title{
Topologically distinct Feynman diagrams for mass operator in electron-phonon interaction
}

\author{
C.C.Tovstyuk* \\ Lviv Polytechnical National University, Institute of Telecommunications Radioelectronics and Electronic \\ Engineering,13 Bandery Str., 79012 Lviv, Ukraine
}

Received February 6, 2009, in final form April 9, 2009

\begin{abstract}
The new method for designing topologically distinct Feynman diagrams for electron's mass operator in electronphonon interaction is developed using the permutation group theory. The carried out classification of DPs allows to choose the classes, corresponding to disconnected diagrams, to singly connected diagrams, direct ("tadpole") diagrams, to diagrams corresponding to phonon Green functions. After this classification the set of considered double permutations is reduced to one class since only these are relevant to mass operator. We derive analytical expressions which allow to identify the DP, and to choose the phonon components, which are not accepted in every type. To avoid repetition of asymmetric diagrams, which correspond to the same analytical expression, we introduce the procedure of inversion in phonon component, and identify symmetric as well as a pair of asymmetric phonon components. For every type of DP (denoted by its digital encoding), taking into account its symmetry, we perform a set of transformations on this DP, list all DPs of the type and all the corresponding Feynman diagrams of mass operator automatically. It is clear that no more expressions (diagrams) for the relevant order of perturbation theory for mass operator can be designed.
\end{abstract}

Key words: Feynman diagrams, electron-phonon interaction

PACS: 71.10.Li, 71.15.Qe, 71.38.Cn, 71.38.-k, 02.20.-a

\section{Introduction}

The Green function methods to be utilized for strong correlated systems come across the problem of convergence of a series and need to take into consideration the high order approximations of perturbation theory, see for example [1]. In [2] all the connected Feynman diagrams for 2 and 3 orders were designed by means of a knot theory for interacting fermions. All Feynman diagrams were designed there, and all disconnected diagrams were dropped. To investigate the mass operator, one has to choose those corresponding to mass operator. The classification of double permutation, introduced here, makes it possible to separate the class of DPs, corresponding to mass operator, and to reduce the set of significant DPs. In the frame of this set, we define different possible types, denoted by their digital encodings, and derive analytical expressions which permit to identify the DP, and to choose the phonon components, which are not accepted in each type. These expressions enable us to find all DPs (Feynman diagrams) for every type automatically for the high orders of perturbation theory. Besides, the pairs of symmetric diagrams, corresponding to the same analytical expression, are identified by introducing the inversion into phonon components and digital encoding. Such a procedure permits to design only Feynman diagrams corresponding to distinct analytical expressions, and to identify the multiplier factor, which denotes this symmetry.

Besides, in literature different numbers of the connected Feynman diagrams are named, they are 84, 3120 in [3], while 74, 706 in [2] for the third and the fourth order of perturbation theory, respectively. Double permutation algebra could be used to find out and prove these exact numbers. Some works devoted to the investigation of mass operator for electron-phonon interaction in nanosystems $[4,5]$ point out the urgency of our investigation. Double permutations, used here, were introduced in [6] and then developed in [7] for interacting electrons.

*E-mail: cornelia@polynet.lviv.ua 
The double permutation is developed for electron-phonon interaction, represented by the Hamiltonian $[8]$

$$
H=\sum_{\alpha \vec{k}} \varepsilon_{\alpha \vec{k}} a_{\alpha \vec{k}}^{+} a_{\alpha \vec{k}}+\sum_{\beta, \vec{q}} \omega_{\beta \vec{q}} b_{\beta \vec{q}}^{+} b_{\beta \vec{q}}+\sum_{\alpha, \beta, \vec{k}, \vec{q}} V_{\alpha \beta}(\vec{q}) a_{\alpha \vec{k}-\vec{q}}^{+} a_{\alpha \vec{k}}\left(b_{\beta-\vec{q}}+b_{\beta \vec{q}}^{+}\right) .
$$

The first term in (1) - is the ideal electron gas Hamiltonian with the energy of $\alpha$-band electron $\varepsilon_{\alpha \vec{k}}$ as a function of electron momentum $\vec{k}$. The second term in $(1)-H_{0}^{\prime}-$ is the ideal phonon gas Hamiltonian with the $\beta$ phonon energy $\left(\omega_{\beta \vec{q}}\right)$, depending on phonon momentum $\vec{q}$. The third term of (1) denotes the interaction between the particles, where $V_{\alpha \beta}(\vec{q})$ is an even interaction function depending on momentum $\vec{q}$. Further, one band approximation is used and indexes $\alpha, \beta$ are omitted.

At $T=0$ the renormalization of electron spectrum is defined by singularity of Fourier transforms of electron Green function, corresponding to Dyson equation:

$$
G(\vec{k}, \omega)=\frac{1}{\omega-\varepsilon-M(\vec{k}, \omega)}
$$

with mass operator $M(\vec{k}, \omega)$, which is defined here using double permutations for the sixth and eighth order of perturbation theory.

Direct method for finding the mass operator of an electron for electron-phonon interaction is developed using the derived expressions for double permutation groups. This method automatically excludes the disconnected and single connected Feynman diagrams. Dependence of electron energy on the momentum is determined from the mass operator. Hence, the shift of the bottom of the conducting band (for semiconductors), the effective mass and the relaxation time of electrons can be defined. Some problems in solid state admit the separation of the terms in infinite series, which are more important (corresponding to the analytical expression with contributions more significant than the other ones). Such expressions convolve to chain integral fractions, and so the mass operator is derived. However, such a convolution can be done only for certain conditions, which essentially depend on the electron and phonon energies as functions of momentum (dispersion laws), and on the binding function $V_{\alpha \beta}(\vec{q})$. Effective interaction of electron gas with acoustical phonons differs from the initial one by an infinitely small value in metals, as it was shown by Migdal [9]. This approximation is used, for example in [10] for electron-phonon interaction in nano-films. Convolution of perturbation series which does not include the effective interaction (crossing dashed lines in Feynman diagrams) to the chain integral fraction was performed in [11]. In [1] the analytic peculiarities of the polaron mass operator in the first two orders of the coupling constant were analyzed and evaluated. It was shown that for some energy values, the contributions of the terms that include an effective interaction should be taken into account. It is also shown that for certain energies, higher orders of perturbation theory are needed. The next important step in finding the electron's mass operator in electron-phonon interaction was made in [12, 13]. The integralfunctional representation of mass operator of quasiparticles interacting with polarization phonons at $T=0$ was derived as the infinite branching integral fraction. The rules outlined in [13] for constructing the diagrams for the mass operator in the higher order of perturbation theory are compared with the results obtained here.

\section{Pair averages and their denoting in double permutation}

Usually the relations between the momenta are derived from Feynman diagrams. These relations define the analytical expression corresponding to each diagram. We define double permutations, and derive the so-called selection rules from them. So, the defined selection rules coincide with expressions of momentum conservation in Feynman diagrams. For Hamiltonian (1) such a double permutation can be formed by substitution in the product $H_{\mathrm{int}}\left(t_{i}\right) H_{\mathrm{int}}\left(t_{i+1}\right)$

$$
\vec{q}_{i+1} \rightarrow-\vec{q}_{i}^{\prime}, \quad \vec{k}_{i+1} \rightarrow \vec{k}_{i}^{\prime}
$$


Then, the product can be written as

$$
\sum_{\beta, \beta^{\prime}} \sum_{k, k^{\prime}} \sum_{q, q^{\prime}} V\left(\vec{q}_{i}\right) V\left(\vec{q}_{i}^{\prime}\right) a_{\vec{k}_{i}-\vec{q}_{i}}^{+} a_{\vec{k}_{i}} a_{\vec{k}_{i}^{\prime}+{\overrightarrow{q^{\prime}}}_{i}}^{+} a_{\vec{k}_{i}^{\prime}} b_{\vec{q}_{i}}^{+} b_{q_{i}^{\prime}} .
$$

In (4), all the creation phonon operators are denoted by non-primed indexes and all the annihilation phonon operators - by primed indexes. The double permutation includes 2 permutations (components), subscribing the electron and phonon pair expectations. The phonon component consists of the top line with digit $i$ (denotes $b_{\vec{q}_{i}}^{+}$) and of the low line digit $j^{\prime}$ (denotes $b_{\vec{q}_{j}^{\prime}}$ ) for pair average $\left\langle T b_{q_{i}}^{+} b_{q_{j}^{\prime}}\right\rangle$. We position the phonon component in the first and in the third line of double permutation. Note: we drop the primes when considering the phonon component out of double permutation. The description of electron pair expectations is located in the first - second, and third - fourth lines of DP in the following way: electron permutation digit $i$ in the first line of DP denotes $a_{\vec{k}_{i}-\vec{q}_{i}}^{+}$and digit $j^{\prime}$ in the third line of DP denotes $a_{\vec{k}_{j}^{\prime}+{\overrightarrow{q^{\prime}}}_{j}}^{+}$. Digits $l, t^{\prime}$ in the second and the fourth lines denote $a_{\vec{k}_{l}}, a_{\vec{k}_{t}^{\prime}}$. Thus, the column of double permutation

$$
\begin{aligned}
& i \\
& l \\
& j^{\prime}
\end{aligned}
$$

denotes the product:

$$
V\left(\vec{q}_{i}\right) V\left(\vec{q}_{j}^{\prime}\right)\left\langle T a_{\vec{k}_{i}-\vec{q}_{i}}^{+} a_{\vec{k}_{l}}\right\rangle \cdot\left\langle T a_{\vec{k}_{j}^{\prime}+\vec{q}_{j}^{\prime}}^{+} a_{\vec{k}_{t}^{\prime}}\right\rangle \cdot\left\langle T b_{\vec{q}_{i}}^{+} b_{\vec{q}_{j}^{\prime}}\right\rangle
$$

with selection rules, according to [7], as follows:

$$
\vec{k}_{i}+\vec{k}_{j}^{\prime}=\vec{k}_{l}+\vec{k}_{t}^{\prime}, \quad \vec{q}_{i}=\vec{q}_{j}^{\prime}=\vec{k}_{i}-\vec{k}_{l}=\vec{k}_{t}^{\prime}-\vec{k}_{j}^{\prime} .
$$

Column (5) corresponds to the element of Feynman diagram, shown in figure 1. From this

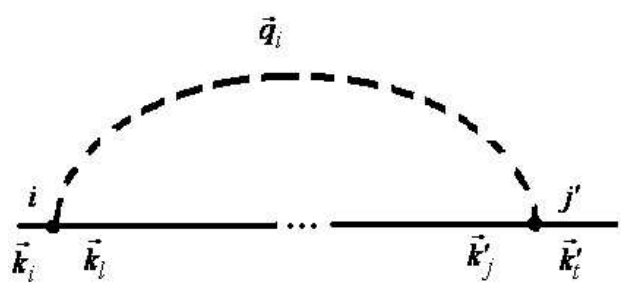

Figure 1. Element of Feynman diagram, corresponding to (5).

element of diagram we get the momentum conservation:

$$
\vec{k}_{l}+\vec{q}_{i}=\vec{k}_{i}, \quad \vec{k}_{j}^{\prime}+\vec{q}_{i}=\vec{k}_{t}^{\prime} .
$$

Expression (8) coincides with the selection rules (7).

\section{Classification of double permutations}

This section provides the classification of double permutations and identifies which class of DP corresponds to disconnected, direct ("tadpole"), singly connected diagrams and Feynman diagrams corresponding to phonon Green function. We also define the class of DP corresponding to mass operator, which is analyzed further. As it is known from the permutation theory [14], permutation group forms classes, which can be represented by Yung schemes and cycles. For DP we form classes after electron component. Each class is partitioned into types after phonon components. Analyzing the structure of DP for different classes and different phonon components we introduce the following classification. 


\subsection{The double permutations, corresponding to disconnected Feynman diagrams}

Analytical expressions of disconnected diagrams include two or more sets of non-bounded momenta. We consider DP with electron component including two (or more) cycles, and phonon component does not assemble any two digits from these cycles. In table 1a we presented such DP, where the vertical line separates parts of different cycles. For such DP, no digit from the first part can be located in the second part. Thus, selection rules (7) are combined from two (or more) sets, which are not connected. Such DPs correspond to disconnected Feynman diagrams, for example as in table $1 \mathrm{a}$, and have to be dropped [8].

Table 1. Different types of Feynman diagrams and peculiarities in corresponding DPs.

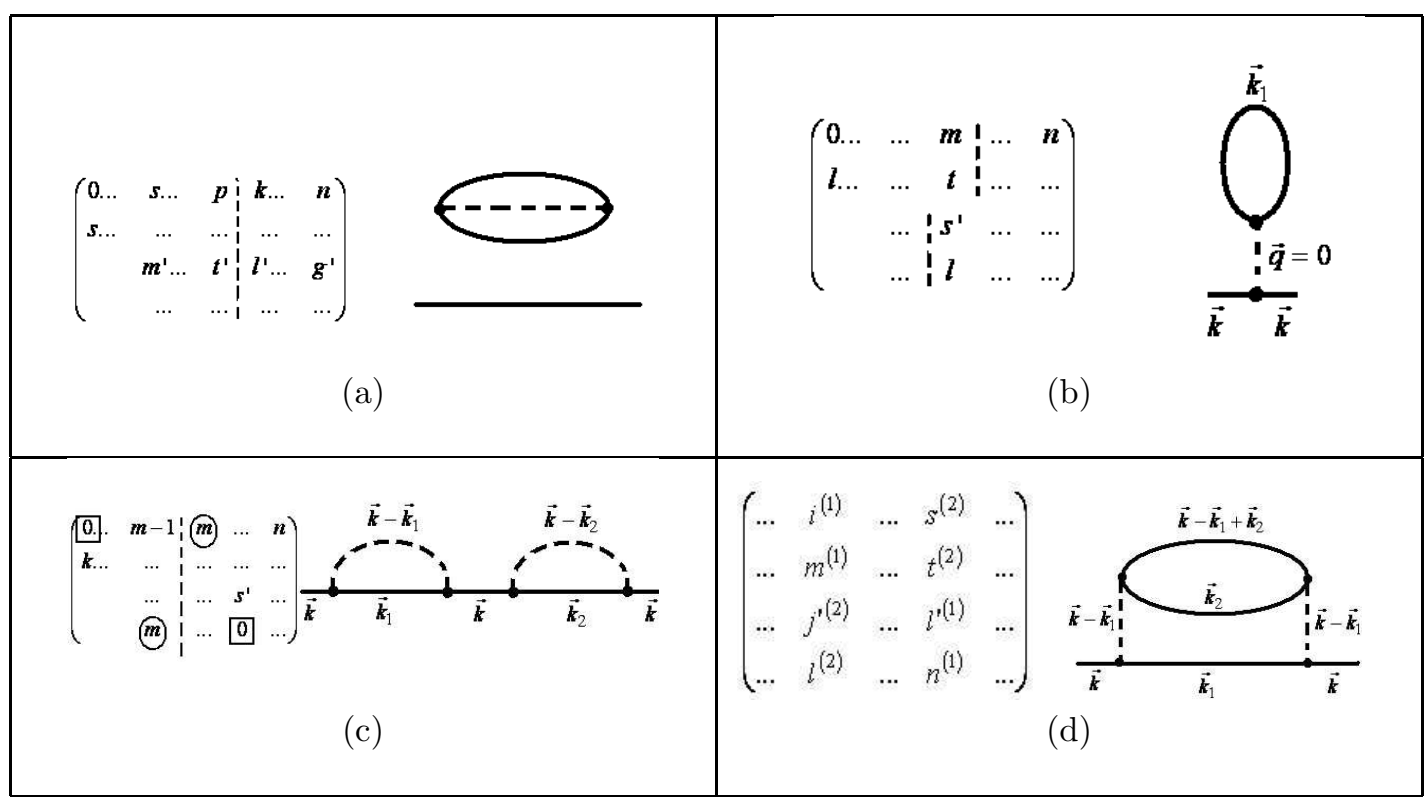

\subsection{The double permutations, corresponding to direct (or "tadpole") Feynman diagrams}

To define such double permutations we choose DP, which includes the electron component with two (or more) cycles and phonon component, which combine only one digit from every cycle. Such $\mathrm{DP}$ is presented in table $1 \mathrm{~b}$ with vertical lines, separating parts of every cycle. In every DP the digits are located two times - once in the first or the third line, once in the second or fourth line. For $i$ - column the selection rules (7) can be written in the form

$$
\vec{k}_{1 i}+\vec{k}_{3 i}-\vec{k}_{2 i}-\vec{k}_{4 i}=0 .
$$

With the first index, denoting the row and the second - denoting the column. The relevant double permutation includes two types of columns: columns with the digits from every cycle, and one combined column ( $m$ in table 1b) which consists of two parts with the digits of the first and the second cycles. Summarizing expressions (9) for the first $m$ columns, we get

$$
\vec{k}_{l}-\vec{k}_{s}^{\prime}=\vec{q}_{m}=0 .
$$

Expression (10) is typical of direct Feynman diagrams, for example as in table 1b.

\subsection{The double permutations, corresponding to singly connected diagrams}

Their analytical expressions define improper self-energy and can be abandoned in self-consistent field methods, like those, related to Dyson's equation [8]. We consider the double permutation 
containing one cycle and phonon component organizing the digits of this cycle into 2 (or more) sets. The relevant double permutation can be presented as it is shown in table 1c. Vertical stroked lines in table 1c separate the parts of DP which correspond to subsets, formed by phonon component. As electron component of DP forms one cycle, every digit from the beginning of every set is located in the second or fourth line from the previous set. Such digits are circled in table 1c. Other digits $(k$; $m$-l;s',n) appear twice in every part of DP. Summarizing the selection rules (9) for all the columns for the last part, we get an additional expression:

$$
\vec{k}_{m}=\vec{k}
$$

which is typical of singly bounded diagrams, for example as in table 1c.

\subsection{The double permutations, corresponding to phonon Green function}

We consider the double permutation which consists of electron component with two cycles and phonon permutation combines two sets of one digit from every cycle. Such DP can be presented as it is shown in table $1 \mathrm{~d}$, where $i, s$ columns are combined. Digits $\left(\ldots i, \ldots m, \ldots l^{\prime}, \ldots n\right)$ form one cycle of electron permutation, and digits $\left(j^{\prime}, \ldots l, \ldots s, \ldots t\right)$ form the other cycle. Summarizing $(9)$ for all columns from the first cycle together with $i, s$ columns, we get

$$
\vec{k}_{j}^{\prime(2)}-\vec{k}_{l}^{(2)}+\vec{k}_{s}^{(2)}-\vec{k}_{t}^{(2)}=0 .
$$

Comparing (12) and (7) we get:

$$
\vec{q}_{i}=\vec{q}_{s}
$$

Equation (13) is typical of Dyson's equation for phonon Green function and corresponding diagram can be shown, for example, as in table $1 \mathrm{~d}$.

To find all Feynman diagrams of electron mass operator only those double permutations are used, which include an electron component with one cycle and phonon component, which form one set of digits.

The Feynman diagram corresponding to DP (for this class), can be constructed using the rule: straight line ("backbone") with $2 n$ nodes and number them in accordance with electron permutations (the first - second and third-fourth rows of DP); phonon lines connect the nodes according to phonon permutation (the first and third rows of DP).

Reassigning the nodes in Feynman diagram we get another DP. It differs from the previous in analytical expression only by silent indexes. To avoid such repetitions, only DP with sub-sets of non-primed and primed numbers arranged in natural order are taken into consideration. Two arranged sub-sets of non-primed and primed numbers can overlap. To follow how they overlap the digital encoding of DP is constructed, which helps to classify double permutations to different types and to avoid the permutations, corresponding to the same diagrams.

\section{Digital encoding and regular double permutation}

Digital encoding is formed for a cycle of electron permutation by denoting all non-primed numbers by 1 and all primed numbers by 0 . Numbers, pointed by 1 denote the start of phonon line and the ones pointed by 0 denote its ending in Feynman diagrams. All possible double permutations of the class are received by listing all phonon components in DP, with the columns accepted for every digital encoding. Then all diagrams, corresponding to DPs can be designed, without their repetitions. We mention here some properties of $\mathrm{DE}$. The first half of DE should not contain less "starts" (1), than "ends" (0), for the second half of DE - this statement is vice versa. To avoid improper diagrams (see 3.3) it is necessary to consider only the cases when phonon lines do not create closed subsets. For DE it means that any part, starting from the beginning, contains more 1-s than 0-s (except the whole DE, which contains the same number of 1 -s and 0 -s). It means 
that for $2 n$ - order of perturbation theory, the digital encoding contains maximum $f$,different" numbers in half of DE ( 0 for the first half and 1 for the second half), which can be defined as:

$$
f=k-1 \text { for } n=\left\{\begin{array}{c}
2 k-1 \\
2 k
\end{array}\right.
$$

Regular double permutation consists of a single cycled electron component with ordered sets of non-primed and primed numbers, which do not overlap. The digital encoding and the cycle of electron component for such DP are:

$$
(11 \ldots 1 \quad 00 \ldots 0) \rightarrow\left(123 \ldots n \quad 1^{\prime} 2^{\prime} 3^{\prime} \ldots n^{\prime}\right)
$$

For unit phonon permutation and (15), regular double permutation looks like:

$$
\left(\begin{array}{lllll}
1 & 2 \ldots & k \ldots & n-1 & n \\
2 & 3 \ldots & k+1 \ldots & n & 1^{\prime} \\
1^{\prime} & 2^{\prime} \ldots & k^{\prime} \ldots & (n-1)^{\prime} & n^{\prime} \\
2^{\prime} & 3^{\prime} \ldots & (k+1)^{\prime} \ldots & n^{\prime} & 1
\end{array}\right)
$$

The type of regular double permutation is formed by listing all the possible phonon permutations for (16). For all corresponding Feynman diagrams of this type the phonon lines are starting in the first half and ending in the second half of backbone (tables 4, 8). This type consists of the largest number of diagrams.

\section{Expressions for columns of phonon permutations and of double permu- tations for digital encodings, different from (15)}

We consider only certain phonon components for digital encodings, different from (15), to avoid the repetition of the same diagrams. The examples of DE, different from (15), together with corresponding cycle of electron component are presented in table 2.

Table 2. Examples of DE, different from (15) and columns of phonon components unaccepted them.

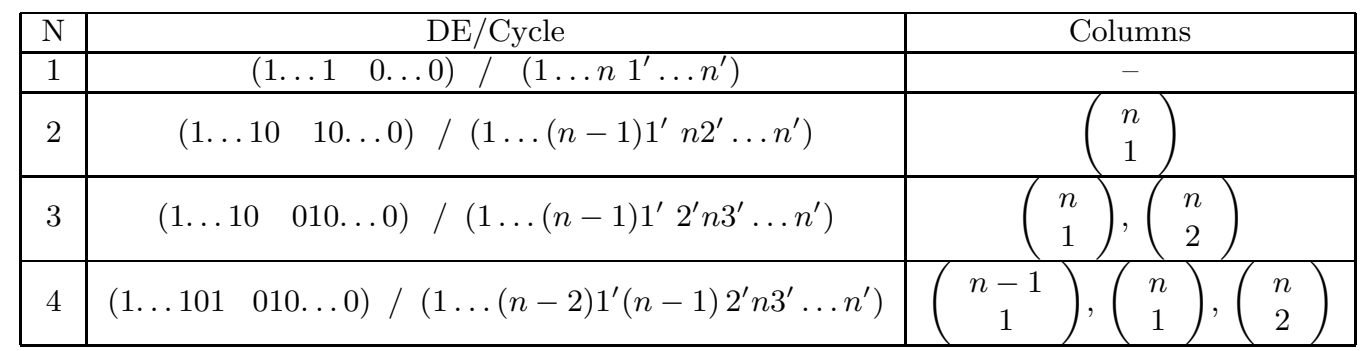

For DE 2 (table 2) the phonon permutations, which combine digits, denoted by 0 from the first part of DE, with the other ones, denoted by 1 from the second part of DE, are dropped (all such combinations were in regular types, besides, the phonon line cannot start from the point, which denotes ending). Thus, phonon components with the columns from table 2 are not accepted. The same statement for DEs 3, 4 (table 2) shows that columns from table 2 are not accepted, to avoid repetitions with previous steps. Let DE contain $t+1$ groups of " 1 " in it. We denote by $k_{i}$ non-primed digits, which follow primed digits and by $l_{i}^{\prime}$ we denote primed digits, which follow non-primed digits in the cycle, as follows:

$$
\begin{aligned}
& (1 \ldots 10 \ldots 01 \ldots 10 \ldots 01 \ldots 10 \ldots 0) \rightarrow \\
& \quad\left(1 \ldots\left(k_{1}-1\right) l_{1}^{\prime} \ldots\left(l_{2}-1\right)^{\prime} k_{1} \ldots\left(k_{i}-1\right) l_{i}^{\prime} \ldots\left(l_{t+1}-1\right)^{\prime} k_{t} \ldots n l_{t+1}^{\prime} \ldots n^{\prime}\right) .
\end{aligned}
$$


The expression which defines unaccepted columns of phonon component for (17) is:

$$
\sum_{i=1}^{t}\left[\left(\begin{array}{l}
k_{i} \\
l_{1}
\end{array}\right) \ldots\left(\begin{array}{l}
k_{i} \\
l_{i+1}-1
\end{array}\right)\right] \ldots\left[\left(\begin{array}{l}
k_{i+1}-1 \\
l_{i}
\end{array}\right) \ldots\left(\begin{array}{l}
k_{i+1}-1 \\
l_{i+1}-1
\end{array}\right)\right], \quad k_{t+1}-1 \equiv n .
$$

In (18), the repetitions of columns are to be neglected. Such repetitions happen if the group of different digits in DE includes only one digit. Double permutation, constructed for unit phonon permutation and (17) is:

$$
\left(\begin{array}{lll}
\ldots k_{i}-1 \ldots & l_{i+1}-1 \ldots & n \\
\ldots l_{i}^{\prime} & \ldots & l_{t+1}^{\prime} \\
\ldots\left(k_{i}-1\right)^{\prime} \ldots & \left(l_{i+1}-1\right)^{\prime} \ldots & n^{\prime} \\
\ldots & k_{i} \ldots & \ldots
\end{array}\right), \quad i=1, \ldots t .
$$

In (19) only digits different from regular DP are placed. For complete expression, the points in (19) are to be replaced by digits from regular DP (16).

\section{Double permutations, corresponding to symmetric and asymmetric dia- grams}

From expressions (19), (18), using DP methodology, topologically distinct diagrams can be designed. Analyzing all the received expressions we see that there are two groups: corresponding to symmetric diagrams, and pairs of expressions corresponding to asymmetric diagrams. The analytical expressions for the pair of asymmetric diagrams are the same. Thus, it is useful to design only one of the pair Feynman diagram, denoting its multiple factor ( 1 for symmetric and 2 for one of the pair of asymmetric ones).

Since the symmetry of a diagram is defined by a phonon component, we performed an operation of inversion for it. We denoted the digits for $2 n$ order of perturbation theory as follows:

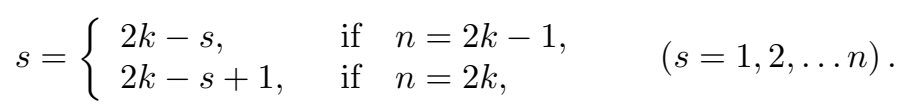

We changed the places of rows and arranged the columns in natural order, according to the properties of permutations [9]. Then, some phonon components do not change - they define symmetric diagrams, and some phonon components change to the other ones - they form a pair of asymmetric diagrams. Thus, the first step in Feynman diagrams design for mass operator is to put down all phonon permutations and to analyze their symmetry about inversion.

Digital encodings, which we use here, for different types, are also symmetric, i. e., those ones, which do not change by denoting 1 to 0 and vise versa and by reading DE goes from right to left; and the pairs of asymmetric DE change from one to another by the same procedure. Analyzing the pairs of asymmetric DE we came to a conclusion that they include pairs of asymmetric diagrams. Thus, it is sufficient to design all the diagrams for one pair of asymmetric DE, and to consider them in mass operator with factor 2 (see, for example table 11).

Symmetric DE includes both, symmetric and pair of asymmetric diagrams. Thus, for types, denoted by symmetric DE we consider the symmetric phonon component with factor 1 and one from the pair of asymmetric component with factor 2 .

Symmetric consideration, subscribed above, allowed us to reduce the number of diagrams from 10,74 to 8,47 in the 6 -th and 8 -th orders of perturbation theory, respectively.

\section{Double permutations and Feyman diagrams for the mass operator in the 6-th order of perturbation theory}

All possible phonon permutations for $n=3$ are presented in table 3 .

Analyzing these permutations about their symmetry according to inversion (see 6 ) we mention that table 3 includes symmetric components $(1,3,5,6)$ and a pair of asymmetric components $(2$ 
Table 3. Phonon components for the 6 -th order of perturbation theory.

\begin{tabular}{|c|c|c|c|c|c|c|c|c|c|c|c|c|c|c|c|c|c|}
\hline \multicolumn{3}{|c|}{1} & \multicolumn{3}{|c|}{2} & \multicolumn{3}{|c|}{3} & \multicolumn{3}{|c|}{4} & \multicolumn{3}{|c|}{5} & \multicolumn{3}{|c|}{6} \\
\hline 1 & 2 & 3 & 1 & 2 & 3 & 1 & 2 & 3 & 1 & 2 & 3 & 1 & 2 & 3 & 1 & 2 & 3 \\
\hline 1 & 2 & 3 & 1 & 3 & 2 & 3 & 2 & 1 & 2 & 1 & 3 & 2 & 3 & 1 & 3 & 1 & 2 \\
\hline
\end{tabular}

- 4). Using (16), the regular DP (1 in table 4) is constructed. The type of regular DP is formed by performing all symmetric and one of the pair of asymmetric phonon components from table 3 . These DPs together with the corresponding Feynman diagrams, their numbers, numbers of phonon components (in parenthesis) and factor [in brackets] are presented in table 4.

Table 4. Regular type of DPs and corresponding Feynman diagrams, their numbers, numbers of phonon components from table 3 (in parenthesis) and factor [in brackets].

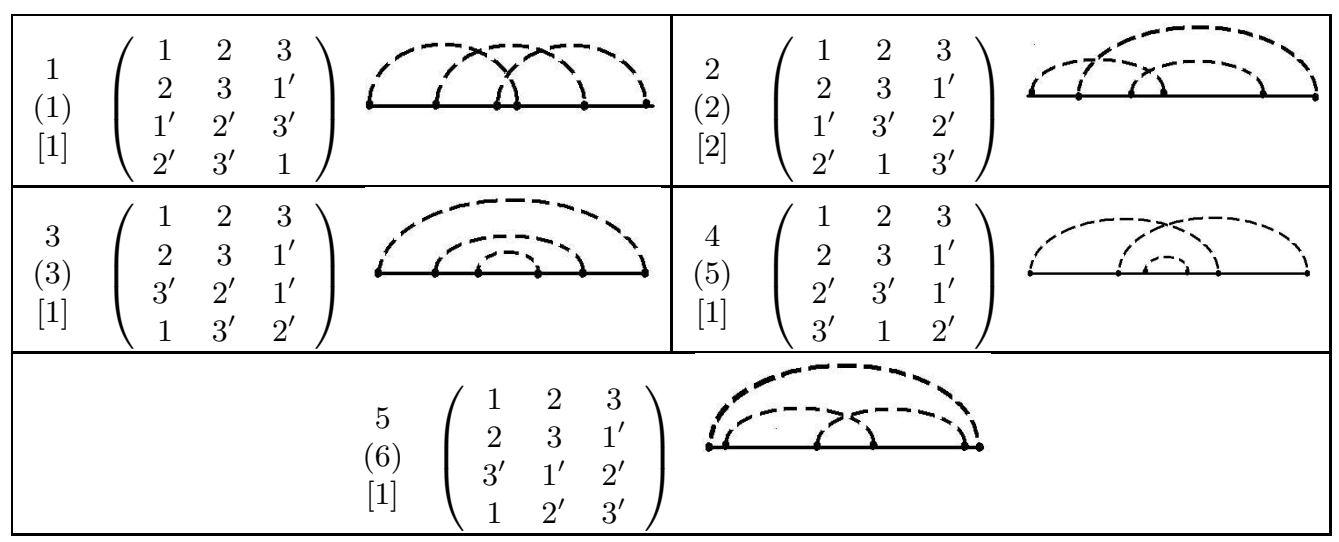

As it follows from (14) only one "different" digit can be in digital encoding. The properties of DE (see 4) make it possible to create only one type of DPs (it is symmetric) for this order. Table 5 includes this DE, parameters, DP, written using (16), column of phonon component, which is not accepted in this type (18), and numbers of accepted phonon component from table 3.

Table 5. DE, parameters, DP (19), column of phonon component, which is not accepted in this type (18), and numbers of accepted phonon component from table 3.

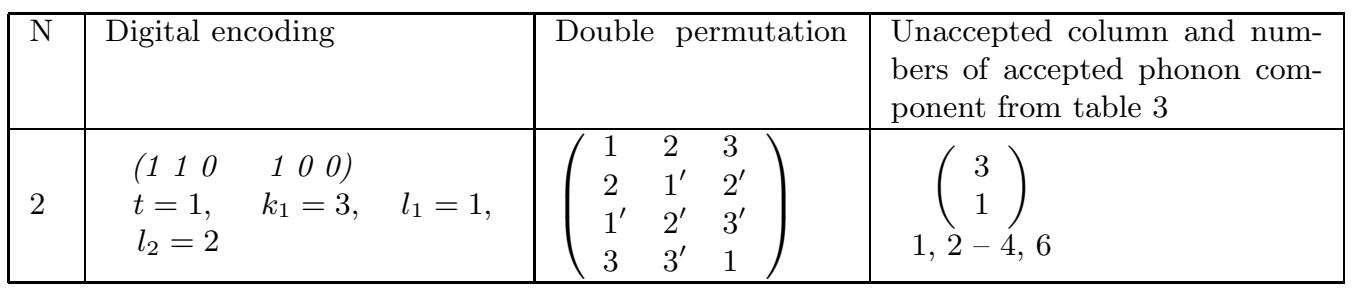

Double permutations and corresponding Feynman diagrams for this type are in table 6 together with their numbers, numbers of the used phonon component (in parenthesis) and factor [in brackets].

Analyzing tables 4 and 6 we see that for cases when the effective interaction differs from the initial one by infinitely small value, only DPs 3 and 8 are to be considered. Other DPs include an effective interaction as follows: DPs 5, 7 include effective interactions in the first order, DPs 1, 2 - include effective interaction of the second order, DP 4 includes effective interaction in the 
Table 6. DP from type 2 table 5, corresponding Feynman diagrams, their numbers, numbers of phonon components from table 3 (in parenthesis) and factor [in brackets].

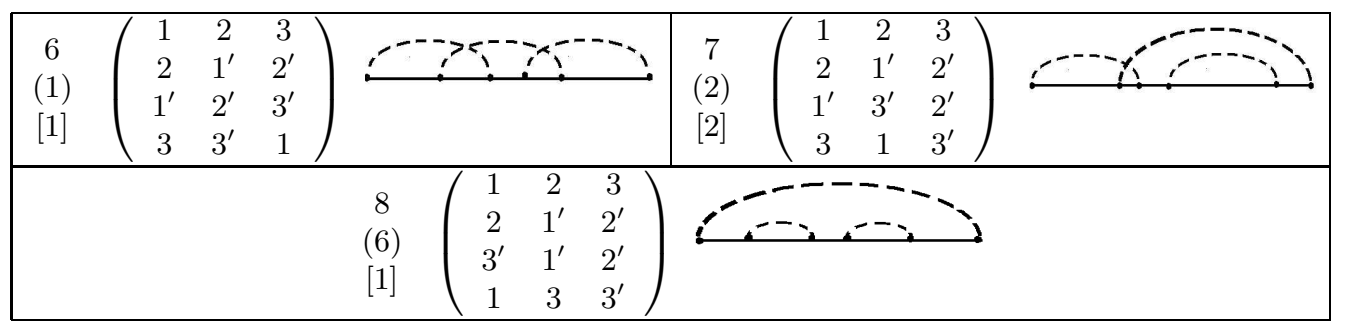

first order with the electron Green function of the first order, DP 6 includes effective interaction in the first order with the effective interaction in the first order. The proper investigation concerning the contributions of DPs 4,6 and 1,2 for polarons in semiconductors are to be carried out. All diagrams from tables 3,5 coincide with the diagrams, presented in [13].

\section{Double permutations and Feynman diagrams for the mass operator in the 8-th order of perturbation theory}

All possible phonon permutations for $n=4$ are listed in table 7 .

Table 7. All possible phonon permutations for $n=4$.

\begin{tabular}{|c|c|c|c|c|c|c|c|c|c|c|c|c|c|c|c|c|c|c|c|}
\hline 1 & 1 & $\begin{array}{l}2 \\
2\end{array}$ & $\begin{array}{l}3 \\
3\end{array}$ & $\begin{array}{l}4 \\
4\end{array}$ & 2 & 1 & $\begin{array}{l}2 \\
2\end{array}$ & $\begin{array}{l}3 \\
4\end{array}$ & $\begin{array}{l}4 \\
3\end{array}$ & 3 & 1 & $\begin{array}{l}2 \\
4\end{array}$ & $\begin{array}{l}3 \\
3\end{array}$ & $\begin{array}{l}4 \\
2\end{array}$ & 4 & 1 & $\begin{array}{l}2 \\
3\end{array}$ & $\begin{array}{l}3 \\
2\end{array}$ & \\
\hline \multirow[b]{2}{*}{5} & 1 & 2 & 3 & 4 & \multirow[b]{2}{*}{6} & 1 & 2 & 3 & 4 & \multirow[b]{2}{*}{7} & 1 & 2 & 3 & 4 & \multirow[b]{2}{*}{8} & 1 & 2 & 3 & 4 \\
\hline & 1 & 3 & 4 & 2 & & 1 & 4 & 2 & 3 & & 4 & 2 & 3 & 1 & & 3 & 2 & 1 & 4 \\
\hline \multirow{2}{*}{9} & 1 & 2 & 3 & 4 & \multirow{2}{*}{10} & 1 & 2 & 3 & 4 & \multirow{2}{*}{11} & 1 & 2 & 3 & 4 & \multirow{2}{*}{12} & 1 & 2 & 3 & 4 \\
\hline & 3 & 2 & 4 & 1 & & 4 & 2 & 1 & 3 & & 2 & 3 & 4 & 1 & & 2 & 4 & 1 & 3 \\
\hline \multirow{2}{*}{13} & 1 & 2 & 3 & 4 & \multirow{2}{*}{14} & 1 & 2 & 3 & 4 & \multirow{2}{*}{15} & 1 & 2 & 3 & 4 & \multirow{2}{*}{16} & 1 & 2 & 3 & 4 \\
\hline & 3 & 4 & 2 & 1 & & 3 & 1 & 4 & 2 & & 4 & 3 & 1 & 2 & & 4 & 1 & 2 & 3 \\
\hline \multirow{2}{*}{17} & 1 & 2 & 3 & 4 & \multirow{2}{*}{18} & 1 & 2 & 3 & 4 & \multirow{2}{*}{19} & 1 & 2 & 3 & 4 & \multirow[b]{2}{*}{20} & 1 & 2 & 3 & 4 \\
\hline & 4 & 3 & 2 & 1 & & 3 & 4 & 1 & 2 & & 2 & 1 & 4 & 3 & & 2 & 4 & 3 & 1 \\
\hline \multirow{2}{*}{21} & 1 & 2 & 3 & 4 & \multirow{2}{*}{22} & 1 & 2 & 3 & 4 & \multirow{2}{*}{23} & 1 & 2 & 3 & 4 & \multirow{2}{*}{24} & 1 & 2 & 3 & 4 \\
\hline & 4 & 1 & 3 & 2 & & 2 & 1 & 3 & 4 & & 2 & 3 & 1 & 4 & & & 1 & 2 & 4 \\
\hline
\end{tabular}

Analyzing the symmetry properties for permutations in table 7 we find out that the permutations symmetric according to inversion are 1,4,7,11,13,15,16,17,18,19; pairs of asymmetric according to inversion permutations are: $2-22,3-8,5-23,6-24,9-20,10-21,12-14$. The regular double permutation (1 in table 8 ) is written, using (16). The type of regular double permutation is formed with all symmetric (with factor 1) and one from the pair of asymmetric phonon components (with factor 2). These DPs together with the corresponding Feynman diagrams, their numbers, numbers of phonon component (in parenthesis) and factor [in brackets] are presented in table 8.

As it follows from (14), maximal number of different digits in half of DE is 1. Table 9 contains all possible digital encodings for $n=4$, (with one from the pair of asymmetric - see 6 ), parameters, DPs (16), not accepted for their types columns of phonon components (18), numbers of accepted phonon components from table 7 .

The DPs and Feynman diagrams for type 2 in table 9 are presented in table 10; for type 3 - in table 11 ; for type 4 - in table 12 .

According to their properties, no more types (DE) can be designed for $n=8$. 
Table 8. Regular type of DPs and corresponding Feynman diagrams, their numbers, numbers of phonon components from table 7 (in parenthesis) and factor [in brackets].

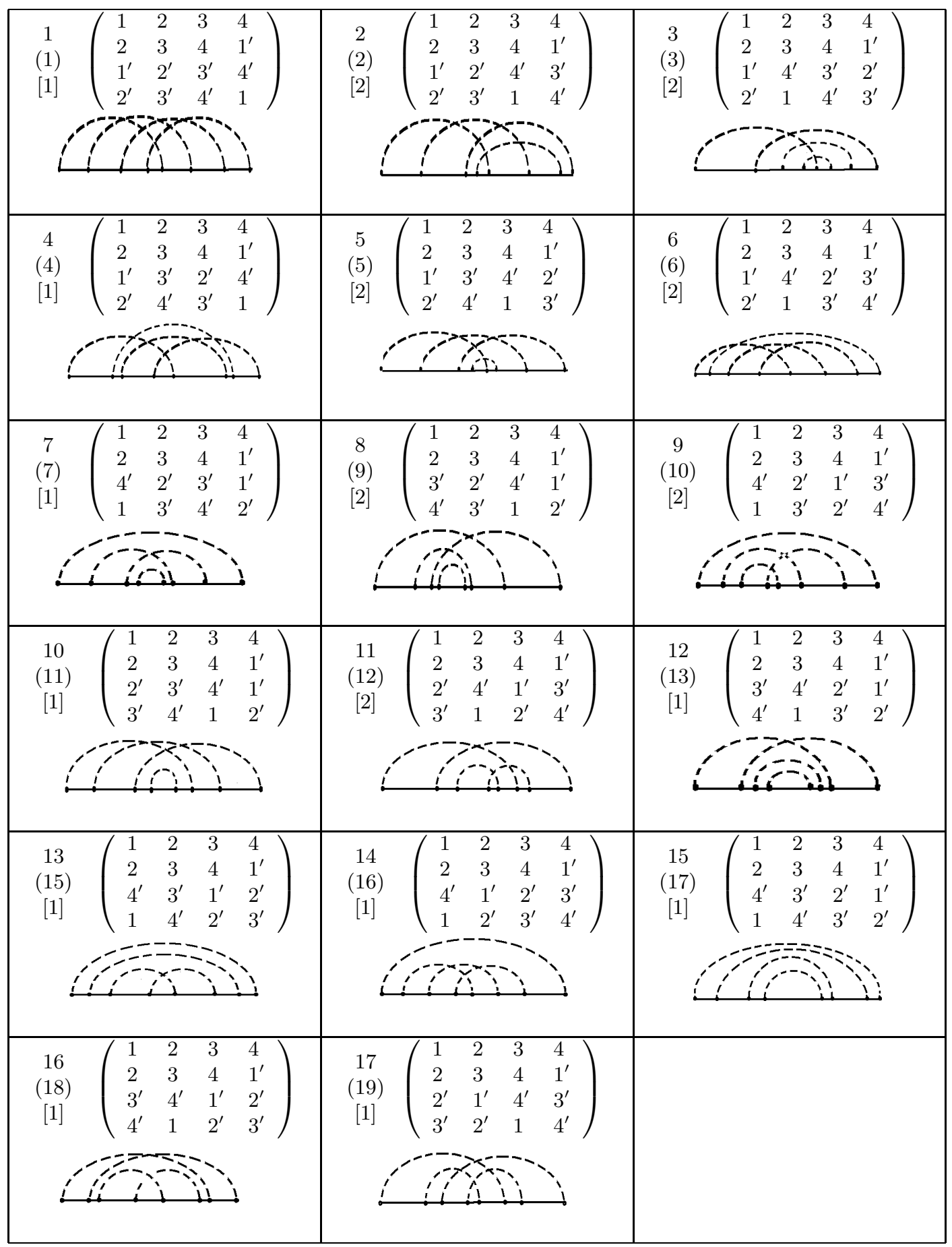

We compared the diagrams from tables 8, 10-12 with the ones constructed using the method proposed in [13]: construction of diagrams for the fourth order, from the diagrams of the third order of perturbation theory (tables 4,6). We obtained the repetitions of the diagrams 39, 19, while diagrams 11 and 17 were not received. Nevertheless, the analytical expression (32) from [13] 
Table 9. Expressions and parameters for the types of DPs with one different digit in the halves of their digital encoding.

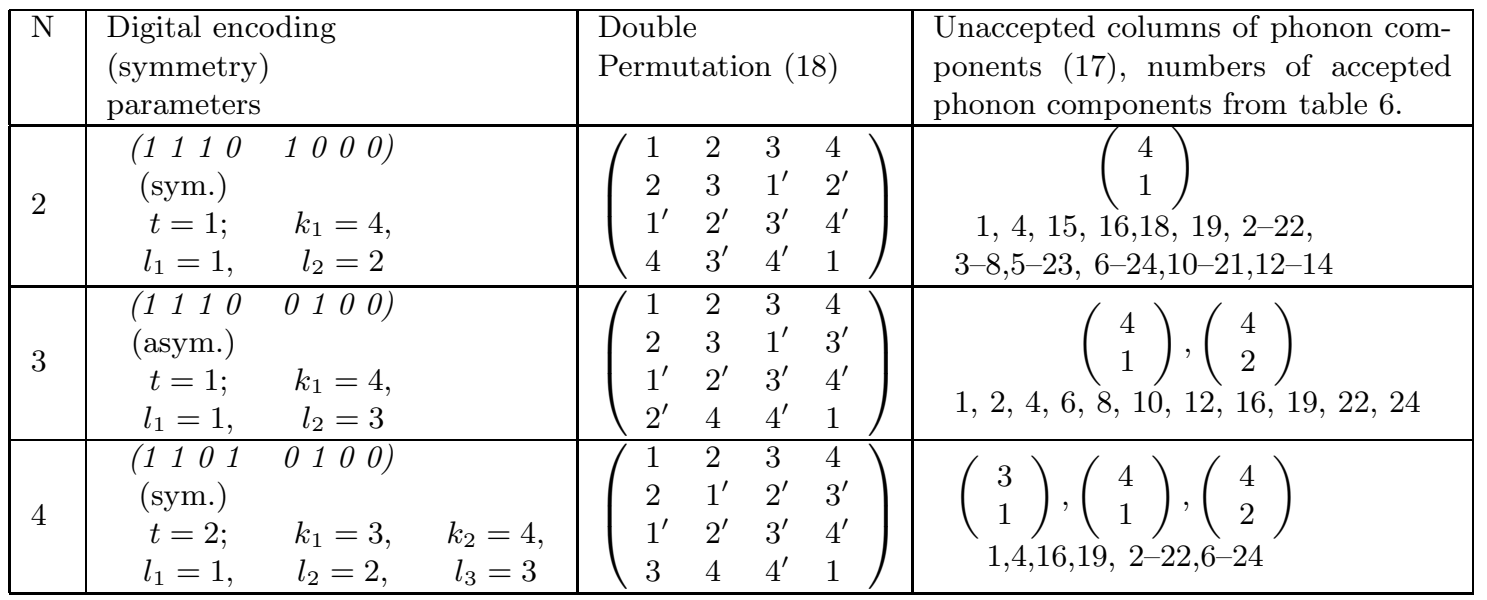

Table 10. DPs type 2 from table 8 and corresponding Feynman diagrams, their numbers, numbers of phonon components From table 7 (in parenthesis) and factor [in brackets].

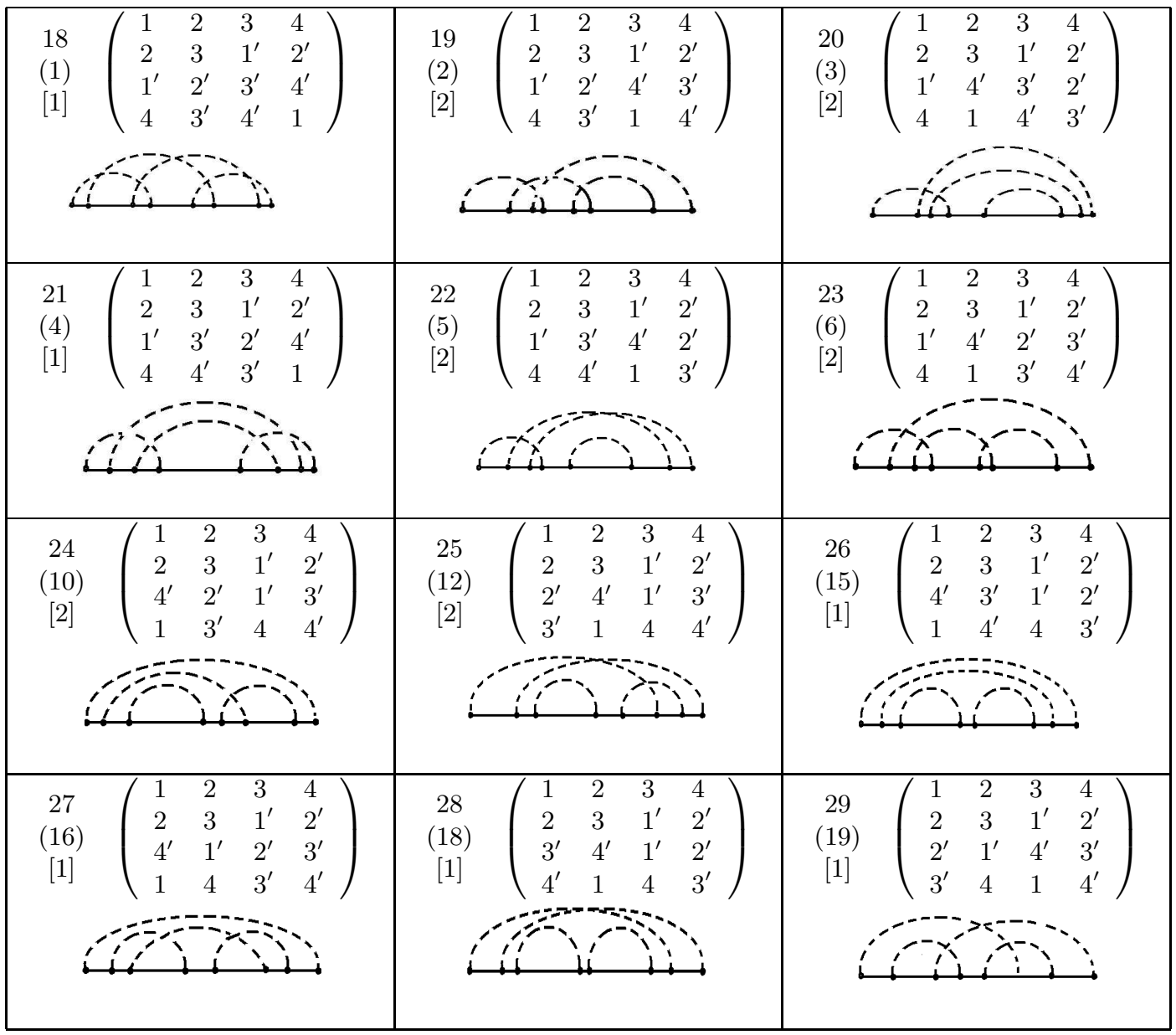


Table 11. DPs type 3 from table 9 and corresponding Feynman diagrams, their numbers, numbers of phonon components From table 7 (in parenthesis) and factor [in brackets].

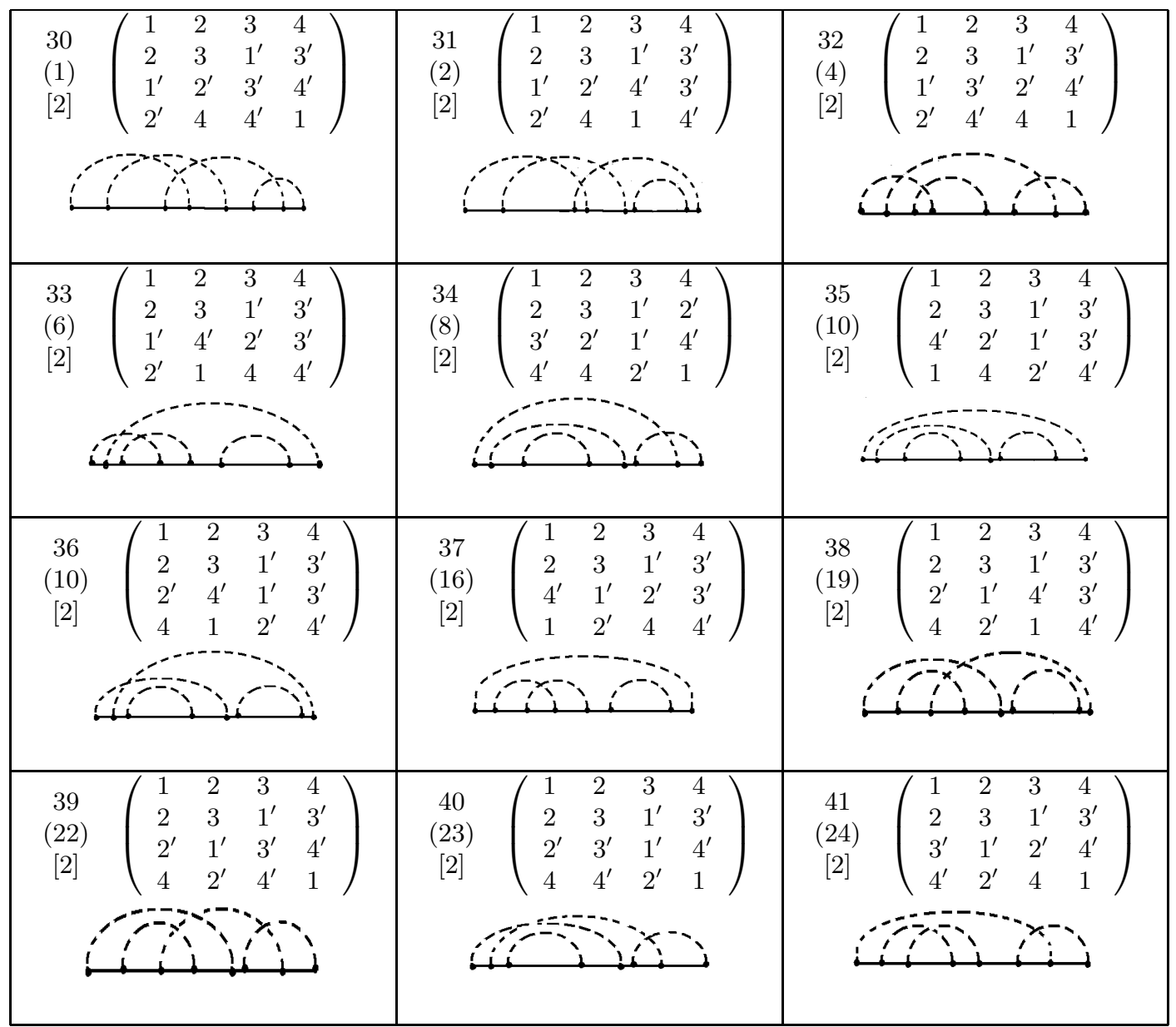

Table 12. DPs type 3 from table 9 and corresponding Feynman diagrams, their numbers, numbers of phonon components From table 7 (in parenthesis) and factor [in brackets].

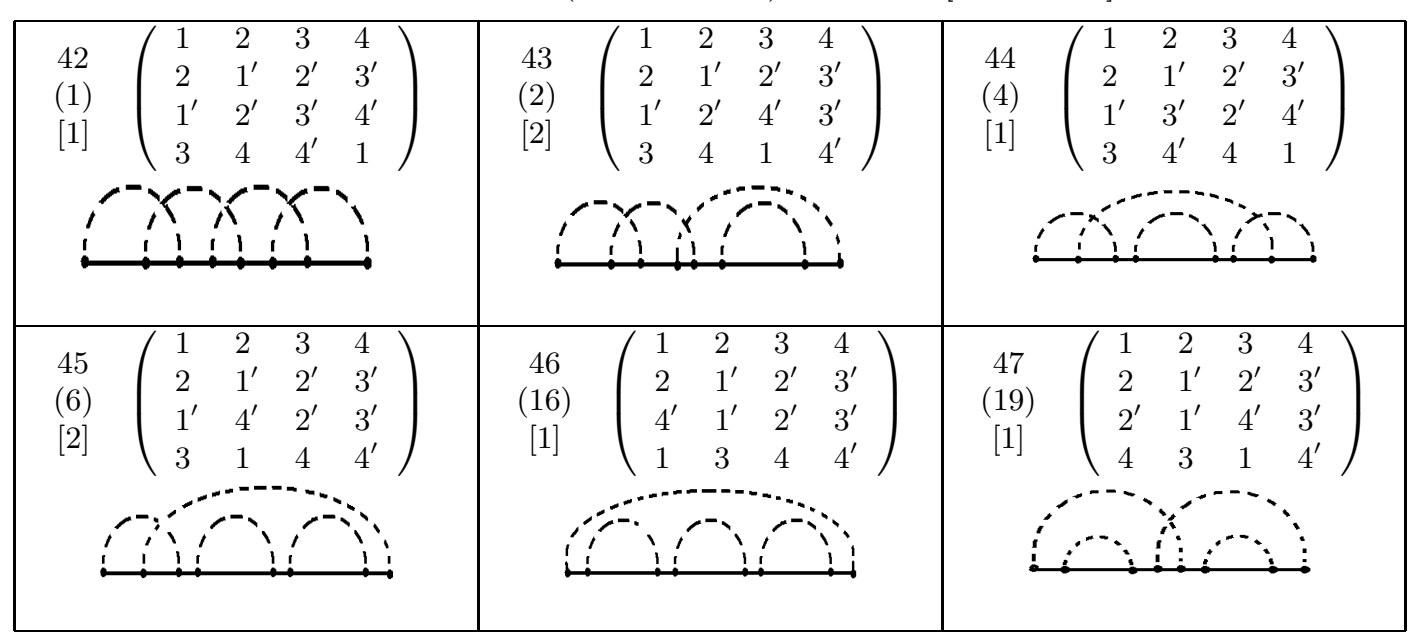


shows that diagrams 11, 17 are included there. So, the proposed method of the construction of the diagrams for the Mass operator, based on double permutation group is more precise, although tables 8, 10-12 include noncompact Feynman diagrams.

\section{Conclusions}

Double permutation method is developed to design all Feynman diagrams for electron mass operator in electron - phonon interaction. The classification of DPs permits to separate the DP, corresponding to disconnected diagrams, to singly connected diagrams, to diagrams corresponding to phonon Green functions. The expressions for DP and unaccepted columns of phonon components were obtained for every type (denoted by its digital encoding). The symmetrical analysis of phonon component and digital encoding is carried out. The derived expression permits to find all Feynman diagrams for mass operator automatically in the higher order of perturbation theory and the symmetry analysis permits to reduce their numbers from 10, 74 to 8,47 for the 6 -th and 8 -th order of perturbation theory.

I appreciate the interest to this work from Prof. M. Tkach, because the discussions with him started this paper.

\section{References}

1. Tkach M., Voitsekivska O., Val O. Condens. Matter Phys., 1998, 1, 401.

2. Mathar R. Int. J. of Quantum Chemistry, 2007, 107, 1975.

3. Cederbaum L.S. Theor. Chim. Acta, 1973, 31, 239.

4. Tkach N.V., Zharkoy V.P. Physics and Technique of Semiconductors, 1999, 33, 598 (in Russian).

5. Tkach N.V. et al. Solid State Physics, 2001, 43, 1315 (in Russian).

6. Tovstyuk K.D. Semiconductor Material Science, "Naukova dumka", Kyjiv, 1984, p.264 (in Russian).

7. Tovstyuk K.D., Tovstyuk C.C., Danylevych O.O. Int. J. of Modern Physics, 2003, B17, 3813.

8. Abrikosov A.A., Gorkov L.P., Dzialoshinski I.E. Methods of quantum field theory in statistical physics. Dover Publ., New York, 1975.

9. Migdal A.B. Journ. of Experim. and Theor. Phys., 1958, 34, 1438 (in Russian).

10. Tkach M.V., Kramar V.M. Ukrainian Journal of Physics, 2008, No. 8, p.812 (in Ukrainian).

11. Pines D. in Polarons and Excitions, Premium Press. N.Y., 1962, p.155-170.

12. Tkach N.V. Theoretical and Mathematical Physics, 1984, 61, No. 3, p.400 (in Russian).

13. Tkach M.V. Journal of Physical Studies, 2002, 6, No. 1, p.124.

14. Kaplan J.G. Symmetry of Many Particles System., Moscow, "Nauka", 1969, p. 435 (in Russian). 


\title{
Топологічно нееквівалентні діаграми Фейнмана для масового оператора при електрон-фононній взаємодії
}

\author{
К.К.Товстюк \\ Національний університет “Львівська політехніка”, Інститут телекомунікацій, радіоелектроніки та \\ електронної техніки, 79012 Львів, вул. С.Бандери 13, Україна
}

Отримано 6 лютого 2009 р., в остаточному вигляді - 9 квітня 2009 р.

\begin{abstract}
Запропоновано новий метод побудови топологічно нееквівалентних діаграм Фейнмана для масового оператора при електрон-фононній взаємодії із використанням методу подвійних перестановок (ПП). Проведено класифікацію ПП, яка дозволяє визначити класи, що відповідають незв'язаним, звідним діаграмам, прямим та діаграмам, які відповідають функції Гріна фононів. Проведена класифікація дозволяє звузити кількість ПП, що розглядається, до одного класу, оскільки саме вони відповідають масовому операторові. Отримано аналітичний вираз, за допомогою якого записуються подвійні перестановки, та обираються фононні компоненти, відсутні для кожного типу. Щоби уникнути записів не симетричних діаграм, які відповідають однаковим аналітичним виразам, вводиться операція інверсії у фононній компоненті і виділяються симетричні та пари несиметричних фононних складових. Для кожного типу ПП (відображеного своїм цифровим зображенням) з врахуванням його симетрії, виконуються описані перетворення ПП, виписуються всі допустимі ПП та відповідні діаграми Фейнмана для масового оператора автоматично. Зрозуміло, що жодних інших виразів (діаграм) для розглянутого наближення теорії збурення не може бути побудовано.
\end{abstract}

Ключові слова: діаграми Фейнмана, електрон-фононна взаємодія

PACS: 02.20.-a, 71.10.Li, 71.15.Qe, 71.38.Cn, 71.38.-k 